\title{
Immunogenicity and safety of a novel monovalent high- dose inactivated poliovirus type 2 vaccine in infants: a comparative, observer-blind, randomised controlled study
}

Authors:

Xavier Sáez-Llorens MD, Hospital del Niño, Ciudad de Panama, Panama.

Ralf Clemens MD, Global Research in Infectious Diseases, Rio de Janeiro, Brasil.

Geert LeRoux Roels MD, Center for Vaccinology (CEVAC), Ghent, Belgium

José Jimeno MD, Vaxtrials, Ciudad de Panama, Panama.

Sue Ann Costa Clemens MD, Instituto de Pós Graduação Carlos Chagas, Rio de Janeiro, Brasil.

William C. Weldon PhD, Centers for Disease Control and Prevention, Atlanta, GA, USA.

M. Steven Oberste PhD, Centers for Disease Control and Prevention, Atlanta, GA, USA.

Natanael Molina PharmD, VaxTrials, Ciudad de Panama, Panama.

Ananda S. Bandyopadhyay MBBS, MPH, Bill \& Melinda Gates Foundation, Seattle, WA, USA. (Corresponding author) 


\section{Summary}

\section{Background:}

Following the proposed global switch from trivalent oral poliovirus vaccine (tOPV) to bivalent types 1 and 3 OPV (bOPV) in 2016, inactivated poliovirus vaccine (IPV) will be the only source of type 2 protection. With most countries opting for one dose of IPV in routine immunisation (RI) schedules during this transition due to cost and manufacturing constraints, optimisation of protection against all polio types will be a priority of the global eradication programme.

\section{Methods:}

We conducted a randomised, controlled trial in infants with a novel monovalent inactivated high-dose type 2 poliovirus vaccine (mIPV2HD) to explore the potential of higher immunogenicity against type 2 with a single dose of inactivated vaccine. This observerblind, comparative, randomised controlled trial in a single center in Panama included healthy infants who had not received any prior vaccination against poliovirus. Infants were randomised into two groups and received single dose of either mIPV2HD or standard trivalent IPV administered concurrently with a third dose of bOPV at 14 weeks of age. At 18 weeks, infants were challenged with monovalent type 2 OPV (mOPV2). Primary endpoints were seroconversion and median antibody titres to type 2 poliovirus 4 weeks after vaccination; and safety (as determined by the incidence and nature of serious adverse events [SAEs] and important medical events [IMEs] for 8 weeks post vaccination) of mIPV2HD/IPV. Secondary endpoints included kinetics of type 2 humoral response; viral shedding following oral type 2 challenge; seroconversion to poliovirus types 1 and 3; and reactogenicity of mIPV2HD/IPV administration along with bOPV. (ClinicalTrials.gov, number NCT02111135). 


\section{Findings:}

Four weeks after vaccination with mIPV2HD/IPV, type 2 humoral immune response was superior in the mIPV2HD group compared to the IPV group. The seroconversion rate was 93.0\% in the mIPV2HD group and $74.8 \%$ in the IPV group (95\% confidence interval [CI] of the difference: $5 \cdot 0-31 \cdot 1 ; \mathrm{p}<0 \cdot 001$ ) and the median type 2 antibody titres were 181 and 36 , respectively (95\% CI of the difference: $60 \cdot 7-136 \cdot 9$; $\mathrm{p}<0 \cdot 0001$ ). As early as 7 days post vaccination, humoral immune response was superior for mIPV2HD. There was no difference between study groups in the proportion of infants shedding type 2 virus or the amount of virus being shed after mOPV2 challenge. SAEs were reported for $6(5 \cdot 1 \%)$ infants in the mIPV2HD group and $7(6 \cdot 0 \%)$ infants in the IPV group during the 8-week post-vaccination period, none were related to vaccination. No IMEs were reported. The solicited reactogenicity profile was similar for the two study vaccines. Seroconversion rates after second dose of bOPV were $92 \cdot 1$ - $87 \%$ for type 1 and $96 \cdot 5-97 \cdot 4 \%$ for type 3 in the two groups that increased to and $95 \cdot 6-91 \cdot 3 \%$ and $\geq 98 \%$ respectively after 3 doses.

\section{Interpretation:}

The superior humoral immunogenicity of mIPV2HD vaccine should support its potential use as an option for stockpiling for outbreak response or primary protection in selected areas at risk for type 2 emergence during the next phase of the polio endgame. Both bOPV-IPV and bOPV-mIPV2HD schedules induced high and comparable seroconversion rates against types 1 and 3 which is reassuring and supports the goal of protection against all types of polio with such new schedules.

Funding: Bill \& Melinda Gates Foundation. 


\section{Introduction}

The world is closer than ever in eradicating polio as the number of cases due to wild type poliovirus (WPV) has decreased dramatically in recent years. ${ }^{1}$ Currently, only two countries, Pakistan and Afghanistan, are considered endemic for polio where WPV transmission has never been interrupted. ${ }^{2}$ Also, of the three serotypes of WPV, only type 1 is currently circulating in these endemic countries. WPV type 2 is considered eradicated as the last naturally occurring case was seen in 1999. However, type 2 Sabin polio virus accounts for approximately 97\% of recent circulating vaccine-derived poliovirus (cVDPV) outbreaks that typically occur in areas with low immunisation coverage and approximately $26-31 \%$ of vaccine-associated paralytic poliomyelitis (VAPP) cases. ${ }^{3}$ No wild type 3 cases have been reported since November 2012, the longest period ever for type 3 circulation interruption. With such historic progress being made in interrupting WPV transmission, estimates suggest that the current burden of vaccine-related poliomyelitis is probably greater than that caused by $\mathrm{WPV}^{1}$. The overall global VAPP risk is estimated to be 4.7 cases per million live births which means an annual incidence of approximately 498 cases. ${ }^{3}$ To add to this, the average number of reported cases of cVDPV has been 76 per year in the recent past ${ }^{1}$. In contrast, the total number of polio cases due to WPV in 2013 and 2014 was 416 and 359, respectively ${ }^{4}$. Therefore, vaccination policies for the endgame need to ensure adequate focus is being given to eliminate all types of polioviruses to achieve and sustain polio eradication for longterm.

With this epidemiologic backdrop, the Polio Eradication and Endgame Strategic Plan was developed by the Global Polio Eradication Initiative (GPEI) in 2013 with the aim of wiping out the last cases of polio from all causes by $2018 .^{5}$ As a first step towards eliminating 
vaccine-related polio disease, the Endgame Plan recommends replacement of tOPV, which protects against types 1, 2 and 3, with bOPV protecting against types 1 and 3 by April 2016 preceded by the introduction of at least one dose of IPV in routine immunisation programmes globally. From 2016 onwards a mixed bOPV-IPV regimen in the Expanded Program on Immunization (EPI) schedule is recommended in which the one dose of IPV would be used with the primary intent to prime the population for immunity against type 2 . In addition, this dose of IPV will boost immunity against types 1 and 3 . The final step of the Endgame Plan would be to stop all OPV use after 2018-2019 and use only IPV for protection against polio. ${ }^{5,6}$ This strategy was endorsed by the Strategic Advisory Group of Experts on immunization (SAGE) in October $2015 .{ }^{7}$

The current formulation of IPV with D-antigen (D-Ag) content of 40, 8, and 32 units for poliovirus types 1,2 , and 3 in IPV stand-alone or combination vaccines was established based on a series of pivotal studies by Salk and his co-workers. ${ }^{8,9,10,11,12}$ Although the formulations containing 320-32-64 and 80-8-16 D-Ag units produced higher seroconversion rates, the 40-8-32 D-Ag unit formulation was chosen because it induced sufficient immune response in infants after administration in full primary series of three or more doses and could be manufactured in adequate quantities. ${ }^{13}$ It has been noted that the immune response from IPV to type 2 virus is low and may be related to its sensitivity to formalin inactivation. ${ }^{14}$ When given on or after 2 months of age, currently available IPV is shown to provide 32-77\% seroconversion against type 2. ${ }^{15,16,17,18}$ Achieving better type 2 protection from a single dose of inactivated vaccine could have significant public health benefit, particularly during the period when tOPV will be replaced by bOPV globally putting type 2 protection at some risk. With the aim to improve type 2 immunogenicity with a single dose, mIPV2HD was formulated in 2013 that contains 32 D-Ag units of type 2 poliovirus, which is four times the type 2 D-Ag units contained in IPV. The type 2 content in this monovalent 
formulation is equivalent to that in the previous experimental 320-32-64 unit trivalent formulation, which was not associated with any safety issues in the initial studies. ${ }^{8,9,10,19}$ The advantage of such a high dose type 2 formulation could be a higher proportion of infants protected with a single dose, and/or better kinetics of priming. Both would be important characteristics for a stockpile vaccine candidate in case of a type 2 outbreak from sources such as undetected circulation of type 2 VDPV after the tOPV-bOPV switch, accidental (or intentional) release from a laboratory or vaccine manufacturer or iVDPV excretion in a population with low coverage. As bOPV has been shown to be more immunogenic than tOPV $^{20}$, the omission of types 1 and 3 from IPV and focus on type 2 response could also allow the use of this vaccine in routine immunisation along with bOPV for a the limited period of time in selected areas at high risk for type 2 emergence.

Although there were a priori no major safety concerns for this mIPV2HD vaccine candidate, the safety profile of this formulation was assessed in a phase I study conducted in 80 healthy adults in Belgium (ClinicalTrials.gov, number NCT01997632). No vaccine-related serious adverse events were reported up to 6 months post-vaccination and treatment-emergent clinically significant abnormal laboratory values were not observed. The reactogenicity profile was similar to that of the control group that received commercially-available IPV.

The phase II trial reported here is the first study of this vaccine in a naïve human population.

\section{Methods}

\section{Study design and participants}

This was an observer-blind, comparative, randomised controlled clinical trial conducted between April 2014 and January 2015 in a single center in Panama. Parents or legal guardians were advised about the trial during the late stage of pregnancy or at the first 
postnatal visit, when the physician assessed eligibility and obtained informed written consent.

Participants were healthy infants approximately 6 weeks (accepted range: 5-8 weeks) of age. Only one infant was enrolled per household. Infants were excluded if they had been previously vaccinated against poliovirus, had a confirmed or suspected immunodeficiency, a low birth weight $(<2,500$ g), or had known allergy to any component of the study vaccines. Infants were also excluded if a household member had received OPV within the previous 3 months or was scheduled to receive OPV during the study period.

Other vaccines were provided according to the national immunisation schedule of Panama to ensure that the infants were fully protected (see legend to Table 5). The study vaccines were thus given concomitantly (but in a different limb) with DTPw-HBV-Hib (or DTPw-Hib followed by Hep B vaccine), pneumococcal conjugate vaccine and oral rotavirus vaccine.

The study was approved by the ethical review board of the Hospital del Niño, Panama, and was conducted in accordance with the Declaration of Helsinki, the International Conference on Harmonisation guideline for Good Clinical Practice, and the codes and regulations of Panama regarding research on human subjects. Oversight of the study was provided by an independent data safety and monitoring board (DSMB).

\section{Randomisation and masking}

Eligible infants were randomised 1:1 into the two study arms using computer-generated randomisation. The randomisation list was maintained concealed from the sponsor, investigators, and the DSMB unless the board ruled otherwise. The study was open-label for the vaccine administrators, but the site staff responsible for safety follow-up (who were different from the vaccinating nurses) and parents or guardians of the study participants were not aware of the treatment group to which the child had been assigned. Laboratory 
personnel responsible for processing and analysing samples and all other assessments were blinded to group allocation.

\section{Procedures}

Infants first received two doses of bOPV (Sanofi Pasteur, Lyon, France) at 6 and 10 weeks of age. At 14 weeks, infants received one intramuscular dose of either mIPV2HD (0.5 ml; Bilthoven Biologicals B.V., Bilthoven, The Netherlands) containing 32 D-Ag units of inactivated type 2 poliovirus or IPV (0.5 ml; Sanofi Pasteur, Lyon, France), which was administered concurrently with a third dose of bOPV. At 18 weeks, all infants were challenged with one dose of mOPV2 (Polio Sabin ${ }^{\mathrm{TM}}$ Mono Two, GlaxoSmithKline, Rixensart, Belgium).

Neutralising antibody titres for poliovirus types 1,2 , and 3 were assessed at $6,14,15,18$ and 19 weeks of age. Serum samples were prepared immediately after collection of blood, stored at $-20^{\circ} \mathrm{C}$, and sent frozen to the Centers for Disease Control and Prevention (CDC, Atlanta, GA) laboratory for analysis using the WHO standard microneutralisation assay, as described previously. ${ }^{18}$ Neutralisation titres were estimated by the Spearman-Kärber method $^{21}$ and expressed as the reciprocal titre of the calculated 50\% endpoint. Titres $>1448$ (the highest dilution tested) were attributed the value 1448. Intestinal shedding of poliovirus type 2 was assessed in stool samples (5 - 10 g/sample) collected using WHO-approved protocols and kits once weekly over a period of three weeks post-mOPV2 challenge; samples were transported frozen to the CDC laboratory for analysis by culture following the WHO protocol. ${ }^{22}$ Type 2 poliovirus titres were expressed as $\log _{10} 50 \%$ cell culture infective dose $\left(\mathrm{CCID}_{50}\right)$ /gram of stool.

Study staff recorded medical history and provided training and a diary card for parents to record safety data and medication use. In addition to filling out the cards, parents/guardians 
were asked at each of the site visits to provide information on any adverse event that occurred since their last visit. A toll-free telephone number was also provided for parents/guardians to call the study team if medical advice was required. Solicited local and general adverse events were recorded for 1 week after administration of study vaccines, unsolicited adverse events (AEs) and concomitant medication for 4 weeks post vaccination, SAEs were recorded over the entire study period of 34 weeks. Important medical events (IMEs), defined as medically significant events which did not meet the criteria for an SAE, but required medical or surgical consultation or intervention to prevent the event from becoming serious, were also recorded over the entire study period. Two blood samples were collected (prior to vaccination and one week after) for routine serum chemistry and haematological laboratory testing.

\section{Outcomes}

Primary immunogenicity outcomes were seroconversion rates and median antibody titres to type 2 poliovirus at 18 weeks of age (i.e., four weeks after vaccination with mIPV2HD or IPV). For infants who were seronegative (titre $<8$ ) at the time of vaccination, seroconversion was defined as achieving an antibody titre $\geq 8$ after vaccination; for infants who were seropositive (titre $\geq 8$ ), seroconversion was defined as having a $\geq 4$-fold rise in titre over expected levels of decay of maternally-derived antibody based on the prevaccination titre (using a half-life of 24 days for maternal antibody). Secondary immunogenicity outcomes included type 2 seroconversion rates and median antibody titres one week after vaccination, quantitative index (percentage of shedders, amount of virus shed, duration of shedding) of type 2 viral shedding over a three-week period following mOPV2 challenge, and the seroconversion rates and median antibody titres for type 1 and 3 poliovirus at 14 and 18 weeks of age. 
The primary safety outcome was the incidence of SAEs and IMEs over the 6-week period after vaccination with mIPV2HD or IPV. Secondary safety outcomes included the incidence of SAEs or IMEs over the entire study; the incidence and severity of solicited local and systemic AEs on the day of vaccination with mIPV2HD/IPV and the following 7 days; incidence of abnormal laboratory values on the day of vaccination with mIPV2HD/IPV and one week later; and incidence and severity of unsolicited AEs from day of vaccination with mIPV2HD or IPV and the following 28 days.

\section{Statistical analysis}

For sample size calculations, type 2 seroconversion rate after one dose of mIPV2HD was considered to be $45 \%$. With a sample size of 108 infants, the power of the study was $90 \%$ to declare superiority of mIPV2HD over IPV if the seroconversion rate was $23 \%$ higher in the mIPV2HD vaccine group than in the IPV group. This sample size also provided $90 \%$ power to declare superiority of mIPV2HD over IPV if the difference in median type 2 neutralisation titres was greater than 9; it also provides a 95\% probability of detecting any safety signal with a true frequency of approximately 1/40, using the rule of three. Assuming that $10 \%$ of the participants would not be evaluable, 120 infants were recruited in each group. The actual retention was adequate for power calculations for primary outcomes.

For the primary objective related to immunogenicity, the 95\% Fisher exact confidence interval (CI) for the difference in binomial proportions was used to assess the superiority of the type 2 seroconversion rate. Superiority was to be concluded if the 95\% CI did not include the null value. Seroconversion rates were also compared using Fisher's exact test. A Hodges-Lehmann estimate, together with the 95\% distribution-free CI (i.e., Moses confidence limit) and a Mann-Whitney-Wilcoxon test, was used to determine whether the difference between the type 2 median neutralisation titres in the two study groups was statistically significant. 
Intestinal immunity to type 2 poliovirus was assessed using the quantitative shedding index ${ }^{18}$, which was based on the average $\log _{10}$-transformed values of virus concentration in stool samples collected at 3 different time points at weekly intervals. Titres of virus shed at each time point and the shedding index were compared between groups using the Mann Whitney-Wilcoxon test.

The primary immunogenicity analyses were performed per intention to treat (ITT), including all subjects for whom a post-vaccination blood sample was available. All subjects were included in the analyses of safety and reactogenicity. Analyses were performed using SAS version 9.2 (SAS Institute, Cary, NC).

\section{Role of the funding source}

The study was funded by the Bill and Melinda Gates Foundation (BMGF). One of the authors of this report (ASB) is an employee of BMGF, and was involved in the study design, data interpretation and writing of the report. All authors had full access to all the data from the study; the corresponding author had the final responsibility for the decision to submit for publication.

\section{Results}

A total of 240 infants were screened; 233 were enrolled in the study between April 14 and May 92014 and randomised to receive mIPV2HD (n=117) or IPV (n=116) (Figure 1). All 233 infants received the allocated polio vaccines at 6 weeks (bOPV), 10 weeks (bOPV) and 14 weeks (bOPV and mIPV2HD or IPV) of age and 230 (98.7\%) infants received the mOPV2 challenge four weeks later at 18 weeks. Overall, $87 \%$ of the randomised infants completed the study. 
Table 1 shows the demographic characteristics of the two groups. The mean age of the infants at enrolment was $6 \cdot 1 \pm 0 \cdot 9$ weeks and the majority was Hispanic (89.6\%). The two groups were well-balanced with respect to gender, breastfeeding and day care attendance.

Approximately $60 \%$ of infants had detectable type 2 maternal antibodies at baseline. This rate fell to $35 \%$ at the time of mIPV2HD/IPV administration at 14 weeks of age. Type 2 seroconversion rates 4 weeks after vaccination (18 weeks of age) were $93 \cdot 0 \%$ (95\% CI: 86.8-96.9) in the mIPV2HD group and $74 \cdot 8 \%$ (95\% CI: 65.8-82.4) in the IPV group. The difference between the 2 groups was $18 \cdot 3 \%$ (95\% CI: $5 \cdot \theta 31 \cdot 1)$, which was statistically significant ( $<<0 \cdot 001)$ (Table 2). The lower bound of the CI of the difference was above 0 , indicating superiority of mIPV2HD. The median type 2 neutralising antibody titre 4 weeks after vaccination (18 weeks of age) was 181 in the mIPV2HD group and 36 in the IPV group; the difference between the 2 groups was also statistically significant (98.8 [95\% CI: 60.7-136.9]; $\mathrm{p}<0 \cdot 001)$.

As shown in Table 2, a significant difference in type 2 seroconversion rate between groups was observed as early as one week after mIPV2HD/IPV vaccination, when it was $93 \cdot 0 \%$ (95\% CI: 86. 8 - 96.9) in the mIPV2HD group and 76.3\% (95\% CI: $67 \cdot 4-83 \cdot 8)$ in the IPV group $(\mathrm{p}<0 \cdot 001)$. The median titres were also higher in the mIPV2HD group compared to the IPV group (288 and 45, respectively) at this time. One week after the mOPV2 challenge, type 2 seroconversion reached $98 \cdot 2 \%$ (95\% CI: $93 \cdot 7$ - 99.8) in the mIPV2HD group compared to $91 \cdot 2 \%(95 \%$ CI: $84 \cdot 5-95 \cdot 7)$ in the IPV group, indicating priming for type 2 from the single dose of inactivated vaccines. 1 of the 2 infants who remained seronegative after vaccination with mIPV2HD and for 7 out of 8 infants who remained seronegative after vaccination with IPV seroconverted within a week of challenge. 
Following mOPV2 challenge, $80(84 \cdot 2 \%)$ infants in the mIPV2HD group and $76(83 \cdot 5 \%)$ infants in the IPV group shed type 2 virus at any time point (Table 3). The shedding index was $3.95 \log _{10} \mathrm{CCID}_{50}$ in the mIPV2HD group and $4.07 \log _{10} \mathrm{CCID}_{50}$ in the IPV group; the distribution of shedding indices in the two groups is shown as reverse cumulative distribution curves in Figure 2 (in Appendix). Virus titres and the proportion of infants shedding virus in each group were highest one week after challenge and diminished thereafter. There were no statistically significant differences between study groups.

At 6 weeks, the percentages of infants with neutralising antibodies were $62 \cdot 3 \%$ and $73 \%$ for type 1 and 36\% and 30.4\% for type 3, respectively for mIPV2HD and IPV groups (Table 4). At 14 weeks of age, i.e., 4 weeks after the second dose of bOPV, seroconversion rates were 92.1\% and $87 \%$ for type 1 and $96 \cdot 5$ and $97 \cdot 4 \%$ for type 3 respectively for mIPV2HD and IPV groups. At 14 weeks, after two doses of bOPV, $\geq 99 \cdot 1 \%$ of the infants had protective antibody levels against types 1 and 3, and median titres were 1448. A third dose of bOPV together with mIPV2HD (no type 1 and 3 polio antigen) or IPV led to all infants being seroprotected with no further increase in titres.

Over the course of the study 8 (6.8\%) infants in the mIPV2HD group and $15(12 \cdot 9 \%)$ infants in the IPV group experienced SAEs; of these, $6(5 \cdot 1 \%)$ infants and 7 (6.0\%) infants, respectively, experienced SAEs during the 8-week period after vaccination with mIPV2HD or IPV (Table 5). None of the SAEs were considered to be related to vaccination. No infant experienced an IME during this period. No clinically relevant differences were apparent in the incidence, nature, or severity of solicited injection site reactions and systemic events between the two vaccine groups. The incidence of infants experiencing other, unsolicited events over the 28-day period after mIPV2HD or IPV vaccination was also similar in the two groups. The causal relationship between the polio vaccines and the systemic reactogenicity cannot be determined with certainty, given that other EPI vaccines were 
administered at the same visit. Virtually all out-of-normal range laboratory values pre- and post-vaccination were considered as without clinical relevance; no change in laboratory parameter was imputed to the study vaccines.

\section{Discussion}

This study is the first to report safety, reactogenicity and immunogenicity of a novel mIPV2HD formulation. It is also an important addition to the clinical evidence base on new polio vaccination schedules with bOPV and IPV that will soon be adopted globally based on the Endgame Strategic Plan. ${ }^{5,23}$ The study demonstrated that one dose of mIPV2HD induces a superior humoral immune response against type 2 in comparison with IPV when given at 14 weeks in the EPI schedule along with the third dose of bOPV, as measured by differences in seroconversion rates and in median titres. Furthermore, the kinetics of the type 2 immune response after the two vaccines was different, with a significantly higher seroconversion rate observed as early as one week after vaccination in infants who received mIPV2HD.

The superior humoral immune response induced by one dose of mIPV2HD vaccine did not translate into improved intestinal immunity compared to standard IPV. After the challenge dose of mOPV2, the percentage of infants who shed virus, the quantity of virus shed, and the duration of shedding did not have any statistically significant difference between the two groups. This finding is consistent with earlier studies that demonstrated the relative lack of primary intestinal immunogenicity from IPV. ${ }^{17,24,25}$

This study also demonstrated excellent humoral immunogenicity induced by two doses of bOPV for types 1 and 3 in the population and environment studied. A third dose of bOPV along with IPV for the IPV group added only marginally to the high response from first two doses. This finding will be important for the global bOPV switch scheduled for April 2016 preceded by the at least one dose IPV introduction. The promising data on priming for type 
2 from one dose of inactivated vaccines (IPV or mIPV2HD) in such schedules indicate potential for rapid immune response to a type 2 exposure in the future. These data also indicate that, for types 1 and 3, bOPV produces very high type 1 and 3 seroconversion rates when administered in an EPI series and thus the lack of protection to these serotypes in the mIPV2HD candidate is not of significant concern, particularly in settings similar to where this study was performed.

The safety profile of the mIPV2HD vaccine was similar to that of the IPV when administered concomitantly in a paediatric vaccination schedule and there was no difference in safety events between the two groups. All SAEs/IMEs reported during the study were events which are routinely observed in children below one year of age and all were considered unrelated to vaccination. There were no clinically relevant differences in injection site events between the two vaccines.

This study had limitations. There was no "OPV-only" or "no polio vaccine” control group. This implies that although there was no difference between the two study groups for intestinal immunogenicity, the impact on shedding compared with no vaccine or OPV administration cannot be assessed. Also, the study was conducted in Panama where tOPV was still being used for RI although no national or regional OPV immunisation campaigns were ongoing during the period that the study was conducted; thus some background exposure of study participants to type 2 Sabin viruses could not be ruled out. However, with the measures taken to minimise this effect, and also the fact that any such effect would have affected both the groups, there is no reason to believe that it had any substantial impact on the study findings and interpretations. Lastly, only the impact of a single dose of mIPV2HD was evaluated; booster dose(s) in the design would have allowed an evaluation of potential additional effects on intestinal mucosal immunity via activation of memory B-cells. Future 
studies will also need to evaluate a potential effect of the mIPV2HD vaccine on concomitantly-administered antigens.

In conclusion, one dose of mIPV2HD was shown to be well-tolerated in infants and to induce a superior humoral immune response, both in terms of magnitude as well as kinetics of the response compared to a single dose of currently available IPV. Based on the promising humoral immunogenicity and safety data from this study, mIPV2HD could be considered an important addition to the polio endgame vaccination options. Also, unlike the live attenuated mOPV2, mIPV2HD does not carry the rare but serious risk of generating new VDPVs or VAPP. On the other hand, the impact of mIPV2HD on primary intestinal immunogenicity was comparable to current IPV. Among other factors, the impact of IPV on intestinal immunity depends on prior exposure to OPV. Two recent studies from India showed that one dose of IPV given to OPV-vaccinated children significantly boosted intestinal mucosal immunity compared to no vaccine and this boost was higher than what was achieved with an additional dose of OPV. ${ }^{26,27}$ Prior studies and reviews have also shown that when IPV was given to children who did not have prior OPV exposure, it had a marginal impact in reducing duration and average titer of faecal viral shedding, which was substantially lower than that induced by OPV. ${ }^{1,18,24}$ Considering all of these factors, mIPV2HD could play an important role in individual protection by closing any humoral immunity gap and would also be effective in boosting intestinal immunity for OPVvaccinated children. But with limited impact on inducing intestinal immunity in naïve children, it is unlikely to be the only tool for large-scale type 2 outbreak control. This new formulation could indeed be stockpiled for concomitant use in outbreak response for control and prevention of type 2 outbreaks following the transition from tOPV to bOPV. In situations where the risk of generating new VDPVs with the use of mOPV2 is considered high in an outbreak response scenario, e.g., in areas of very poor immunisation coverage, 
mIPV2HD could also be an alternative to mOPV2 as it does not have any risk of VAPP or VDPV generation. This may also apply to areas surrounding the epicentre of an outbreak. Enhanced environmental surveillance to rapidly detect any silent virus circulation after such use of inactivated vaccines in these areas would add value to the overall response strategy. Considering the supply and cost constraints of IPV ${ }^{5,28}$, mIPV2HD could be an alternative to IPV for primary protection against type 2 during the period following OPV2 withdrawal and before all OPV cessation. However, such benefits have to be balanced with issues such as lack of any type 1 and 3 protection compared to IPV, need for an accelerated pathway for licensure for RI use and the challenges of multiple short-term changes in immunisation programs before moving to all IPV by 2019. Whilst the safety and immunogenicity data of this candidate vaccine look promising additional information such as immunogenicity in different age groups and with multiple doses, concomitant use with mOPV2 for outbreak control, and eventually alternative needle-free administration methods for easier use in outbreak response settings would further support informed policy decisions for polio vaccination for the endgame and beyond. 


\section{Contributors}

All authors reviewed and approved the manuscript. XSL was the principal investigator of the study. ASB and RC participated in the development of the study design, interpretation of the data and reviewed the study report. JJ and NM participated in the study design, conduct and reviewed the study report. SACC provided important input into the study design and logistical aspects of the trial. WCW and MSO supervised the laboratory analyses and interpreted the laboratory data. GLR was the principal investigator of the first study evaluating the mIPV2HD vaccine (IPV-004) and provided support in the preparation of the manuscript. 


\section{Acknowledgments}

The findings and conclusions in this report are those of the author(s) and do not necessarily represent the views of CDC and other contributing agencies. The use of trade names is for identification purposes only and does not constitute an endorsement by the Centers for Disease Control and Prevention or the US Government.

Important contributors were: Ana Cecilia Villarreal who was the site coordinator for the study; Steve Self, Bhavesh R. Borate and Chris Gast of the Fred Hutchinson Cancer Research Center, who provided input into study design and data analysis, respectively; Chris Wilson of the Bill \& Melinda Gates Foundation who provided input into study design; and Mohamed Amakrane, Marie-Cécile Bozonnat and Anne Hepburn of 4Clinics, who performed the statistical analysis and assisted in the preparation of the study report and manuscript.

The authors wish to thank Victor Sales and Johnny Escobar of VaxTrials for their support in the study conduct; Mark Pallansch, Centers for Disease Control and Prevention, for his expert advice during the development of the study design; María Luisa Ávila, Maria Teresa Valenzuela, Claudio Strunchiner, Luiza Helena Falleiros, and Carolina Danovaro for study supervision within the Data Safety Monitoring Board; Gerrit Van Roekel and Kim Bush of the Bill \& Melinda Gates Foundation for coordinating vaccine supply; Jay Wenger and John Modlin of the Bill \& Melinda Gates Foundation who provided input into study design and data analysis; and the laboratory staff at the Centers for Disease Control and Prevention (Deborah Moore, Yiting Zhang, Sharla McDonald, Larin McDuffie, William Hendley, Patricia Mitchell, Mario Nicolas, Demetrius Mathis, Brittani Brown and Jessica Wielgus) for performing poliovirus titrations and seroneutralisation assays. We also thank Bilthoven Biologicals, GlaxoSmithKline and Sanofi Pasteur for kindly donating vaccines for the study. We are grateful to the Panama Site members Juan Carlos Batista, Luis Casal, Evelyn 
Castillo, Rita Tello, María Guadalupe de Fletcher, John Solano, Onix Saldaña and Stephany Álvarez for their participation. 


\section{Research in Context}

\section{Evidence before this study}

In 2012, to phase-out the use of live type 2 poliovirus vaccine, WHO’s Strategic Advisory Group of Experts on Immunization (SAGE) recommended that trivalent oral polio vaccine (tOPV) be replaced by bivalent OPV (bOPV) containing only poliovirus types 1 and 3 in all countries by 2016. This change is to be preceded by the introduction of at least one dose of conventional trivalent inactivated poliovirus vaccine (IPV) in routine immunisation programmes to provide immunity to type 2 . However, a dose of inactivated type 2 higher than the standard dose (8 D-Ag units) may be needed to ensure adequate immunity. With the aim of improving type 2 immunogenicity with a single dose, a new monovalent high-dose inactivated poliovirus vaccine (mIPV2HD), which contains four times the standard dose of inactivated type 2 poliovirus (32 D-Ag units), was formulated. Higher D-Ag content of IPV was investigated by Salk and co-workers with a series of dose ranging studies several decades ago and literature searches with "IPV”, "high-dose”, “poliovirus”, and/or “type 2" revealed a number of published reports of clinical trials investigating high-dose IPV formulated from inactivated Sabin strains of poliovirus. However, we are not aware of any other published study in which a monovalent high dose inactivated vaccine was compared with trivalent IPV in a mixed bOPV-IPV schedule, and where mOPV2 was used to assess intestinal immunity in such schedules.

\section{Added value of this study}

We are the first to report data on safety and humoral and intestinal immunogenicity of mIPV2HD formulation in a naïve infant population using a mixed bOPV-IPV schedule. The humoral immune response to type 2 with mIPV2HD was superior to that of IPV and the intestinal immunity to type 2 poliovirus was similar in both groups. These results provide 
evidence that mIPV2HD can be safely used in infants and show that a combined bOPVmIPV2HD schedule would adequately protect against all three poliovirus types.

\section{Implications of all the available evidence}

With the upcoming global switch from tOPV to bOPV, the only protection against type 2 will come from IPV. Considering the supply and cost constraints of IPV, higher immunogenicity against type 2 from a single dose of a monovalent high dose formulation could be of advantage in settings at risk of type 2 emergence. Our study established excellent safety and immunogenicity profile of mIPV2HD compared to currently available IPV and therefore is an important addition to the clinical evidence base for the polio endgame vaccine options. 


\section{References}

1 Bandyopadhyay AS, Garon J, Seib K, Orenstein WA. Polio vaccination: past, present and future. Future Microbiol 2015; 10(5): 791-808.

2 World Health Organization. Global Polio Eradication Initiative. Polio this week. Geneva, Switzerland: World Health Organization; 2015. Available at http://www.polioeradication.org/Dataandmonitoring/Poliothisweek.aspx.

3 Platt LR, Estívariz CF, Sutter RW. Vaccine-associated paralytic poliomyelitis: A review of the epidemiology and estimation of the global burden. J Infect Dis 2014; 210(suppl 1): S380-89.

4 Polio Global Eradication Initiative. Polio Eradication \& Endgame; Midterm Review July $2915 . \quad$ Available at: http://www.polioeradication.org/Portals/0/Document/Resources/StrategyWork/GPEIMTR_July2015.pdf

5 Global Polio Eradication Initiative. Polio Eradication \& Endgame Strategic Plan 2013-2018 [Internet]. (2013). Available from: http://www.polioeradication.org/Portals/0/Document/Resources/StrategyWork/PEES P_EN_US.pdf.

6 World Health Organization (WHO). Polio vaccines: WHO position paper, January 2014. Wkly Epidemiol Rec 2014; 89: 73-92.

7 SAGE confirms global polio vaccine switch date as April 2016. 2015. Available at http://www.polioeradication.org/mediaroom/newsstories/SAGE-confirms-globalpolio-vaccine-switch-date-as-April2016/tabid/526/news/1307/Default.aspx?popUp=true 
8 Salk J, Cohen H, Fillastre C, et al. Killed poliovirus antigen titration in humans. Dev Biol Stand 1977; 41: 119-32.

9 Salk J, Van Wezel AL, Stoeckel P, et al. Theoretical and practical considerations in the application of killed poliovirus vaccine for the control of paralytic poliomyelitis. Dev Biol Stand 1980; 47: 181-98.

10 Salk J, Stoeckel P, van Wezel AL, Lapinleimu K, van Steenis G. Antigen content of inactivated poliovirus vaccine for use in a one- or two-dose regimen. Ann Clin Res 1982; 14: 204-12.

11 Vidor E, Mescheivitz C, Plotkin S. Fifteen years of experience with Vero-produced enhanced potency inactivated poliovirus vaccine. Ped Infect Dis J 1997; 16: 312-22.

12 Swartz TA, Handsher R, Stoeckel P, Drucker J, Caudrelier P, Van Wezel AL, Cohen H, Salk D, Salk J. Immunologic memory induced at birth by immunization with inactivated polio vaccine in a reduced schedule. Eur J Epidemiol 1989 5(2): 143-5.

13 World Health Organization (WHO) Technical Report Series 673 Annex 2. Requirements for poliomyelitis vaccine (inactivated). 1982; 40-84.

14 Ferguson M, Wood DJ, Minor PD. Antigenic structure of poliovirus in inactivated vaccines. J GenVirol 1993; 74: 685-90.

15 Simasathien S, Migasena S, Beuvery C, Vansteenis G, Samakoses R, et al. Comparison of enhanced potency inactivated poliovirus vaccine (eIPV) versus standard oral poliovirus vaccine (OPV) in thai infants. Scand J Infect Dis 1994; 26: 731-8. 
16 Mohammed AJ, AlAwaidy S, Bawikar S, Kurup PJ, Elamir E, et al. Fractional doses of inactivated poliovirus vaccine in Oman. $N$ Engl J Med 2010; 362: 2351-9.

17 Resik S, Tejeda A, Sutter RW, et al. Priming after a fractional dose of inactivated poliovirus vaccine. $N$ Engl J Med 2013; 368(5): 416-24.

18 O’Ryan Gallardo M, Bandyopadhyay AS, Villena R, et al. Inactivated poliovirus vaccine given alone or in a sequential schedule with bivalent oral poliovirus vaccine in Chilean infants: a randomised, controlled, open-label, phase 4, non-inferiority study. Lancet Infect Dis 2015; 15(11): 1273-82.

19 Report from WHO Expert Committee on Biological Standardization [Internet]. World Health Organization, Geneva. Available from: http://whqlibdoc.who.int/trs/WHO_TRS_673.pdf.

20 Sutter RW, John TJ, Jain H, et al. Immunogenicity of a bivalent types 1 and 3 oral poliovirus vaccine: a randomised, double-blind, controlled trial. Lancet 2010; 376: 1682-8.

21 Karber G. Beitrag zur kollektiven Behandlung pharmakologischer Reihenversuche. Archiv fur Experimentelle Pathologie und Pharmakologie 1931; 162: 480-3.

22 World Health Organization (WHO). Polio laboratory manual, $4^{\text {th }}$ edition. 2004. (WHO/IVB/04.10)

23 Sutter RW, Bahl S, Deshpande JM, et al. Immunogenicity of a new routine vaccination schedule for global poliomyelitis prevention: an open-label, randomised controlled trial. Lancet 2015; Sep 17. pii: S0140-6736(15)00237-8. doi: 10.1016/S0140-6736(15)00237-8. 
24 The Cuba IPV Study Collaborative Group. Randomized, placebo-controlled trial of inactivated poliovirus vaccine in Cuba. New Engl J Med 2007; 356: 1536-44.

25 Hird TR, Grassly NC. Systematic review of mucosal immunity inducted by oral and inactivated poliovirus vaccines against virus shedding following oral poliovirus shedding. Plos Pathogens 2012; 8(4): e1002599.

26 John J, Giri S, Karthikeyan AS, et al. Effect of a single inactivated poliovirus vaccine dose on intestinal immunity against poliovirus in children previously given oral vaccine: an open-label, randomised controlled trial. Lancet 2014; 384(9953): 150512.

27 Jafari H, Deshpande JM, Sutter RW, et al. Polio eradication. Efficacy of inactivated poliovirus vaccine in India. Science 2014; 345(6199): 922-5.

28 Global Polio Eradication Initiative (GPEI). Availability and price of inactivated polio vaccine. Available at: http://www.polioeradication.org/tabid/488/iid/354/Default.aspx 


\section{Figure 1: Trial profile}

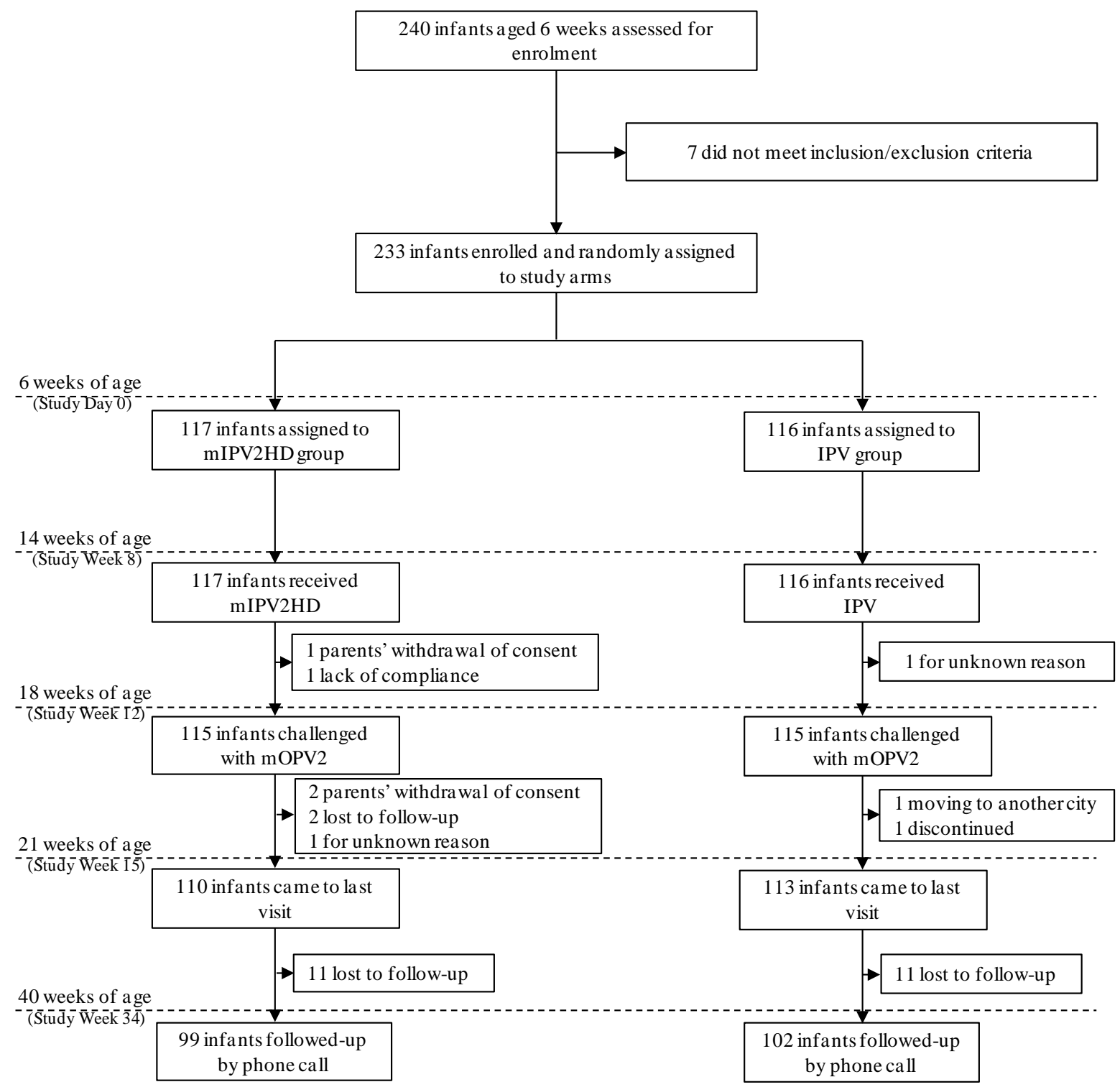

mIPV2HD $=$ monovalent inactivated poliovirus vaccine, type 2 , high dose. IPV $=$ trivalent inactivated poliovirus vaccine. mOPV2 = monovalent oral poliovirus vaccine, type 2 .

Polio vaccines were administered on at 6 weeks (bOPV), 10 weeks (bOPV) and 14 weeks of age (bOPV and mIPV2HD or IPV). Infants received the mOPV2 challenge at 18 weeks of age. 
Table 1: Characteristics of the Intent to treat population

\begin{tabular}{|c|c|c|c|c|}
\hline & & mIPV2HD group & IPV group & Total \\
\hline Number of infants & & 115 & 115 & 230 \\
\hline Age (weeks) & Mean (SD) & $6 \cdot 0(0 \cdot 84)$ & $6 \cdot 1(0 \cdot 92)$ & $6 \cdot 1(0 \cdot 88)$ \\
\hline \multirow[t]{2}{*}{ Gender } & Male & $57(49 \cdot 6 \%)$ & $63(54 \cdot 8 \%)$ & $120(52 \cdot 2 \%)$ \\
\hline & Female & $58(50 \cdot 4 \%)$ & $52(45 \cdot 2 \%)$ & $110(47 \cdot 8 \%)$ \\
\hline \multirow{5}{*}{$\begin{array}{l}\text { Geographic } \\
\text { ancestry }\end{array}$} & White/Caucasian & $2(1 \cdot 7 \%)$ & $1(0 \cdot 9 \%)$ & $3(1 \cdot 3 \%)$ \\
\hline & Black or African American & $5(4 \cdot 3 \%)$ & $10(8 \cdot 7 \%)$ & $15(6 \cdot 5 \%)$ \\
\hline & Asian & 0 & 0 & 0 \\
\hline & Hispanic & $105(91 \cdot 3 \%)$ & $101(87 \cdot 8 \%)$ & $206(89 \cdot 6 \%)$ \\
\hline & Other & $3(2 \cdot 6 \%)$ & $3(2 \cdot 6 \%)$ & $6(2 \cdot 6 \%)$ \\
\hline \multirow{2}{*}{$\begin{array}{l}\text { Child being } \\
\text { breastfed }\end{array}$} & Yes & $112(97 \cdot 4 \%)$ & $111(96 \cdot 5 \%)$ & $223(97 \cdot 0 \%)$ \\
\hline & No & $3(2 \cdot 6 \%)$ & $4(3 \cdot 5 \%)$ & $7(3 \cdot 0 \%)$ \\
\hline \multirow{2}{*}{$\begin{array}{l}\text { Child attending a } \\
\text { day care center }\end{array}$} & Yes & $1(0 \cdot 9 \%)$ & 0 & $1(0 \cdot 4 \%)$ \\
\hline & No & $114(99 \cdot 1 \%)$ & 115 (100\%) & $229(99 \cdot 6 \%)$ \\
\hline
\end{tabular}

Data are mean (SD) or n (\%). mIPV2HD = monovalent inactivated poliovirus vaccine, type 2, high dose. IPV

$=$ trivalent inactivated poliovirus vaccine. 
Table 2: Seroprotection rates, seroconversion rates and median neutralising antibody titres for type 2 poliovirus

\begin{tabular}{|c|c|c|c|c|c|c|c|}
\hline \multirow[b]{2}{*}{ Age (weeks) } & & \multicolumn{2}{|c|}{ mIPV2HD group } & \multicolumn{2}{|c|}{ IPV group } & \multicolumn{2}{|c|}{ Difference } \\
\hline & & $\mathbf{n} / \mathbf{N}$ & $\begin{array}{c}\% \\
(95 \% \mathrm{CI}) \dagger\end{array}$ & $\mathbf{n} / \mathbf{N}$ & $\begin{array}{c}\% \\
(95 \% \mathrm{CI}) \dagger\end{array}$ & $\begin{array}{c}\text { value } \\
(95 \% \mathrm{CI})\end{array}$ & P-value* \\
\hline 6 & SP & $71 / 114$ & $\begin{array}{c}62 \cdot 3 \\
(52 \cdot 7-71 \cdot 2)\end{array}$ & $68 / 115$ & $\begin{array}{c}59 \cdot 1 \\
(49 \cdot 6-68 \cdot 2)\end{array}$ & - & - \\
\hline 14 & SP & $44 / 115$ & $\begin{array}{c}38 \cdot 3 \\
(29 \cdot 4-47 \cdot 8)\end{array}$ & $39 / 115$ & $\begin{array}{c}33 \cdot 9 \\
(25 \cdot 3-43 \cdot 3)\end{array}$ & - & - \\
\hline \multirow[t]{3}{*}{15} & SP & $114 / 115$ & $\begin{array}{c}99 \cdot 1 \\
(95 \cdot 3-100 \cdot 0)\end{array}$ & $108 / 114$ & $\begin{array}{c}94 \cdot 7 \\
(88 \cdot 9-98 \cdot 0) \\
\end{array}$ & - & - \\
\hline & SC & $107 / 115$ & $\begin{array}{c}93 \cdot 0 \\
(86 \cdot 8-96 \cdot 9)\end{array}$ & $87 / 114$ & $\begin{array}{c}76 \cdot 3 \\
(67 \cdot 4-83 \cdot 8)\end{array}$ & $\begin{array}{c}16 \cdot 7 \\
(3 \cdot 7-29 \cdot 1)\end{array}$ & $<0 \cdot 001$ \\
\hline & Median titre $\left(\right.$ dil $\left.^{-1}\right)$ & - & $\begin{array}{c}288 \\
(90 \cdot 5-910 \cdot 2)\end{array}$ & - & $\begin{array}{c}45 \\
(18 \cdot 0-144 \cdot 0)\end{array}$ & - & - \\
\hline \multirow[t]{3}{*}{18} & SP & $113 / 115$ & $\begin{array}{c}98 \cdot 3 \\
(93 \cdot 9-99 \cdot 8) \\
\end{array}$ & $106 / 114$ & $\begin{array}{c}93 \cdot 0 \\
(86 \cdot 6-96 \cdot 9) \\
\end{array}$ & - & - \\
\hline & SC & $107 / 115$ & $\begin{array}{c}93 \cdot 0 \\
(86 \cdot 8-96 \cdot 9) \\
\end{array}$ & & $\begin{array}{c}74 \cdot 8 \\
(65 \cdot 8-82 \cdot 4) \\
\end{array}$ & $\begin{array}{c}18 \cdot 3 \\
(5 \cdot 0-31 \cdot 1) \\
\end{array}$ & $<0 \cdot 001$ \\
\hline & Median titre $\left(\right.$ dil $\left.^{-1}\right)$ & & $\begin{array}{c}181 \\
(72 \cdot 0-362 \cdot 0)\end{array}$ & & $\begin{array}{c}36 \\
(18 \cdot 0-113 \cdot 8)\end{array}$ & $\begin{array}{c}98 \cdot 8 \\
(60 \cdot 7-136 \cdot 9)\end{array}$ & $<0 \cdot 0001$ \\
\hline \multirow[t]{4}{*}{19} & SP & $111 / 112$ & $\begin{array}{c}99 \cdot 1 \\
(95 \cdot 1-100 \cdot 0)\end{array}$ & $113 / 114$ & $\begin{array}{c}99 \cdot 1 \\
(95 \cdot 2-100 \cdot 0)\end{array}$ & - & - \\
\hline & SC & $110 / 112$ & $\begin{array}{c}98 \cdot 2 \\
(93 \cdot 7-99 \cdot 8)\end{array}$ & $104 / 114$ & $\begin{array}{c}91 \cdot 2 \\
(84 \cdot 5-95 \cdot 7)\end{array}$ & $\begin{array}{c}7 \cdot 0 \\
(-6 \cdot 4-19 \cdot 9)\end{array}$ & $0 \cdot 0338 *$ \\
\hline & SC* & $1 / 2$ & $\begin{array}{c}50.0 \\
(1 \cdot 3-98 \cdot 7)\end{array}$ & $7 / 8$ & $\begin{array}{c}87.5 \\
(47 \cdot 3-99 \cdot 7)\end{array}$ & - & - \\
\hline & Median titre $\left(\right.$ dil $\left.^{-1}\right)$ & - & $\begin{array}{c}362 \\
(144 \cdot 0-910 \cdot 2)\end{array}$ & - & $\begin{array}{c}181 \\
(56 \cdot 9-455 \cdot 1)\end{array}$ & - & - \\
\hline
\end{tabular}

mIPV2HD = monovalent inactivated poliovirus vaccine, type 2 , high dose. IPV = trivalent inactivated poliovirus vaccine.

$\mathrm{n}=$ number of infants seroprotected/seroconverted; $\mathrm{N}=$ total number of infants at each time point

$\mathrm{SP}=$ seroprotection (neutralising titre $\geq 8$ )

$\mathrm{SC}=$ seroconversion (with respect to status at 14 weeks of age). For infants who were seronegative (titre $<8$ ), seroconversion was defined as achieving an antibody titre $\geq 8$; for infants who were seropositive (titre $\geq 8$ ), seroconversion was defined as having a $\geq 4$-fold rise in titre over expected levels of decay of maternally-derived antibody based on the pre-vaccination titre. Seroconversion rates were compared using Fisher's exact test.

SC* = seroconversion in infants seronegative at 18 weeks of age. Seroconversion was defined as achieving an antibody titre $\geq 8$.

$\mathrm{CI}=$ confidence interval

$\dagger$ range first to third quartile for median titre; median neutralising antibody titres were compared using the Mann Whitney-Wilcoxon test. * exploratory analysis 
Table 3: Quantitative viral shedding for type 2 virus following mOPV2 challenge

\begin{tabular}{|c|c|c|c|c|}
\hline Age (weeks) & & mIPV2HD group & IPV group & P-value \\
\hline \multirow[t]{2}{*}{$\begin{array}{l}19 \\
\text { (1 week post-challenge) }\end{array}$} & $\begin{array}{l}\mathrm{n} / \mathrm{N} \\
\%(95 \% \mathrm{CI})\end{array}$ & $\begin{array}{c}88 / 108 \\
81 \cdot 5 \%(72 \cdot 9-88 \cdot 3)\end{array}$ & $\begin{array}{c}83 / 106 \\
78 \cdot 3 \%(69 \cdot 2-85 \cdot 7)\end{array}$ & $0 \cdot 6109 *$ \\
\hline & $\begin{array}{l}\text { Median (log) } \text { CCID }_{50} \\
(\mathrm{Q} 1-\text { Q3) }\end{array}$ & $\begin{array}{c}4 \cdot 86 \\
(2 \cdot 84-6 \cdot 20)\end{array}$ & $\begin{array}{c}4 \cdot 45 \\
(2 \cdot 75-6 \cdot 22)\end{array}$ & $0 \cdot 4740^{*}$ \\
\hline \multirow[t]{2}{*}{$\begin{array}{l}20 \\
\text { (2 weeks post-challenge) }\end{array}$} & $\begin{array}{l}\mathrm{n} / \mathrm{N} \\
\%(95 \% \mathrm{CI})\end{array}$ & $\begin{array}{c}67 / 108 \\
62 \cdot 0 \%(52 \cdot 2-71 \cdot 2)\end{array}$ & $\begin{array}{c}64 / 105 \\
60 \cdot 9 \%(50 \cdot 9-70 \cdot 3)\end{array}$ & $0 \cdot 8888 *$ \\
\hline & $\begin{array}{l}\text { Median (log) } \text { CCID }_{50} \\
(\mathrm{Q} 1-\mathrm{Q} 3)\end{array}$ & $\begin{array}{c}2 \cdot 83 \\
(2 \cdot 75-4 \cdot 83)\end{array}$ & $\begin{array}{c}3 \cdot 06 \\
(2 \cdot 75-4 \cdot 72)\end{array}$ & $0 \cdot 8173^{*}$ \\
\hline \multirow[t]{2}{*}{$\begin{array}{l}21 \\
\text { (3 weeks post-challenge) }\end{array}$} & $\begin{array}{l}\mathrm{n} / \mathrm{N} \\
\%(95 \% \mathrm{CI})\end{array}$ & $\begin{array}{c}46 / 101 \\
45 \cdot 5 \%(35.6-55.8)\end{array}$ & $\begin{array}{c}46 / 100 \\
46 \cdot 0 \%(36 \cdot 0-56 \cdot 3)\end{array}$ & $1 \cdot 0000^{*}$ \\
\hline & $\begin{array}{l}\text { Median (log) } \text { CCID }_{50} \\
(\mathrm{Q} 1-\mathrm{Q} 3)\end{array}$ & $\begin{array}{c}2 \cdot 75 \\
(2 \cdot 75-3 \cdot 47)\end{array}$ & $\begin{array}{c}2 \cdot 75 \\
(2 \cdot 75-4 \cdot 47)\end{array}$ & $0 \cdot 5365 *$ \\
\hline Viral shedder at any timepoint & $\begin{array}{l}\mathrm{n} / \mathrm{N} \\
\%(95 \% \mathrm{CI})\end{array}$ & $\begin{array}{c}80 / 95 \\
84 \cdot 2 \%(75 \cdot 3-90 \cdot 9)\end{array}$ & $\begin{array}{c}76 / 91 \\
83 \cdot 5 \%(74 \cdot 3-90 \cdot 5)\end{array}$ & $1 \cdot 0000$ \\
\hline Shedding Index† & $\begin{array}{l}\text { Median (log) } \mathrm{CCID}_{50} \\
(\mathrm{Q} 1-\mathrm{Q} 3)\end{array}$ & $\begin{array}{c}3 \cdot 95 \\
(3 \cdot 18-4 \cdot 85)\end{array}$ & $\begin{array}{c}4 \cdot 07 \\
(3 \cdot 21-4 \cdot 84)\end{array}$ & $0 \cdot 6429$ \\
\hline
\end{tabular}

mIPV2HD = monovalent inactivated poliovirus vaccine, type 2, high dose. IPV = trivalent inactivated poliovirus vaccine.

$\mathrm{n}=$ number of infants shedding virus; $\mathrm{N}=$ total number of infants at each time point

† computed as the average of $\log _{10}$-transformed values of viral titres measured in stool collected on 7, 14, and 21 days post-mOPV2 challenge

The proportions of viral shedders were compared using Fisher's exact test. Viral titres were compared using the Mann Whitney-Wilcoxon test. * exploratory analysis 
Table 4: Seroprotection rates, seroconversion rates and median neutralising antibody titres for type 1 and type 3 poliovirus

\begin{tabular}{|c|c|c|c|c|c|}
\hline \multirow[b]{2}{*}{$\begin{array}{c}\text { Age } \\
\text { (weeks) }\end{array}$} & & \multicolumn{2}{|c|}{ mIPV2HD group } & \multicolumn{2}{|c|}{ IPV group } \\
\hline & & $\mathbf{N}$ & $\begin{array}{c}\% \\
(95 \% \mathrm{CI})\end{array}$ & $\mathbf{N}$ & $\begin{array}{c}\% \\
(95 \% \mathrm{CI})\end{array}$ \\
\hline \multicolumn{6}{|c|}{ Type 1} \\
\hline \multirow[t]{2}{*}{6} & SP & 114 & $\begin{array}{c}62 \cdot 3 \\
(52 \cdot 7-71 \cdot 2)\end{array}$ & \multirow[t]{2}{*}{115} & $\begin{array}{c}73 \cdot 0 \\
(64 \cdot 0-80 \cdot 9)\end{array}$ \\
\hline & Median titre $\left(\right.$ dil $\left.^{-1}\right)$ & & $\begin{array}{c}11 \cdot 31 \\
(5 \cdot 66-36 \cdot 00)\end{array}$ & & $\begin{array}{c}18 \cdot 00 \\
(7 \cdot 11-90 \cdot 51)\end{array}$ \\
\hline \multirow[t]{3}{*}{14} & $\mathrm{SP}$ & 115 & $\begin{array}{c}99 \cdot 1 \\
(95 \cdot 3-100 \cdot 0)\end{array}$ & \multirow[t]{3}{*}{115} & $\begin{array}{c}99 \cdot 1 \\
(95 \cdot 3-100 \cdot 0)\end{array}$ \\
\hline & SC & & $\begin{array}{c}92 \cdot 1 \\
(85 \cdot 5-96 \cdot 3)\end{array}$ & & $\begin{array}{c}87 \cdot 0 \\
(79 \cdot 4-92 \cdot 5)\end{array}$ \\
\hline & Median titre $\left(\right.$ dil $\left.^{-1}\right)$ & & $\begin{array}{c}1448 \\
(1448-1448)\end{array}$ & & $\begin{array}{c}1448 \\
(1448-1448)\end{array}$ \\
\hline \multirow[t]{3}{*}{18} & SP & 115 & $\begin{array}{c}100 \cdot 0 \\
(96 \cdot 8-100 \cdot 0)\end{array}$ & \multirow[t]{3}{*}{115} & $\begin{array}{c}100 \cdot 0 \\
(96 \cdot 8-100 \cdot 0)\end{array}$ \\
\hline & SC & & $\begin{array}{c}95 \cdot 6 \\
(90 \cdot 1-98 \cdot 6)\end{array}$ & & $\begin{array}{c}91 \cdot 3 \\
(84 \cdot 6-95 \cdot 8)\end{array}$ \\
\hline & Median titre $\left(\right.$ dil $\left.^{-1}\right)$ & & $\begin{array}{c}1448 \\
(1448-1448)\end{array}$ & & $\begin{array}{c}1448 \\
(1448-1448)\end{array}$ \\
\hline \multicolumn{6}{|c|}{ Type 3} \\
\hline \multirow[t]{2}{*}{6} & SP & 114 & $\begin{array}{c}36 \cdot 0 \\
(27 \cdot 2-45 \cdot 5)\end{array}$ & \multirow[t]{2}{*}{115} & $\begin{array}{c}30 \cdot 4 \\
(22 \cdot 2-39 \cdot 7)\end{array}$ \\
\hline & Median titre $\left(\right.$ dil $\left.^{-1}\right)$ & & $\begin{array}{c}5 \cdot 66 \\
(5 \cdot 66-14 \cdot 22)\end{array}$ & & $\begin{array}{c}5 \cdot 66 \\
(5 \cdot 66-11 \cdot 31)\end{array}$ \\
\hline \multirow[t]{3}{*}{14} & SP & 115 & $\begin{array}{c}100 \cdot 0 \\
(96 \cdot 8-100 \cdot 0)\end{array}$ & \multirow[t]{3}{*}{115} & $\begin{array}{c}99 \cdot 1 \\
(95 \cdot 3-100 \cdot 0) \\
\end{array}$ \\
\hline & SC & & $\begin{array}{c}96.5 \\
(91 \cdot 3-99 \cdot 0)\end{array}$ & & $\begin{array}{c}97 \cdot 4 \\
(92 \cdot 6-99 \cdot 5)\end{array}$ \\
\hline & Median titre (dil-1) & & $\begin{array}{c}1448 \\
(1152 \cdot 06-1448)\end{array}$ & & $\begin{array}{c}1448 \\
(910 \cdot 17-1448)\end{array}$ \\
\hline \multirow[t]{3}{*}{18} & SP & 115 & $\begin{array}{c}100 \cdot 0 \\
(96 \cdot 8-100 \cdot 0)\end{array}$ & \multirow[t]{3}{*}{115} & $\begin{array}{c}100 \cdot 0 \\
(96 \cdot 8-100 \cdot 0)\end{array}$ \\
\hline & SC & & $\begin{array}{c}98 \cdot 2 \\
(93 \cdot 8-99 \cdot 8)\end{array}$ & & $\begin{array}{c}98 \cdot 3 \\
(93 \cdot 9-99 \cdot 8)\end{array}$ \\
\hline & Median titre (dil-1) & & $\begin{array}{c}1448 \\
(1448-1448)\end{array}$ & & $\begin{array}{c}1448 \\
(1448-1448)\end{array}$ \\
\hline
\end{tabular}

mIPV2HD = monovalent inactivated poliovirus vaccine, type 2, high dose. IPV = trivalent inactivated poliovirus vaccine.

$\mathrm{SP}=$ seroprotection (seroneutralising titre $\geq 8$ ), $\mathrm{SC}=$ seroconversion (with respect to status at 6 weeks of age). For infants who were seronegative (titre $<8$ ), seroconversion was defined as achieving an antibody titre $\geq 8$; for infants who were seropositive (titre 8), seroconversion was defined as having a $\geq 4$-fold rise in titre over expected levels of decay of maternally-derived antibody based on the pre-vaccination titre. 
Table 5: Adverse events after vaccination with mIPV2HD or IPV

\begin{tabular}{|c|c|c|c|c|c|}
\hline & \multicolumn{2}{|c|}{ mIPV2HD group } & \multicolumn{2}{|c|}{ IPV group } & \multirow{2}{*}{ 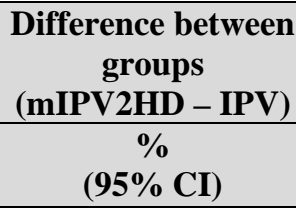 } \\
\hline & $\mathbf{n}$ & $\begin{array}{c}\% \\
(95 \% \mathrm{CI})\end{array}$ & $\mathbf{n}$ & $\begin{array}{c}\% \\
(95 \% \mathrm{CI})\end{array}$ & \\
\hline Number of infants vaccinated & 117 & & 116 & & \\
\hline Any solicited adverse event within 1 week after mIPV2HD/IPV vaccination & 107 & $\begin{array}{c}91 \cdot 5 \\
(84 \cdot 8-95 \cdot 8)\end{array}$ & 112 & $\begin{array}{c}96 \cdot 6 \\
(91 \cdot 4-99 \cdot 1)\end{array}$ & $\begin{array}{c}-5 \cdot 1 \\
(-17 \cdot 8-7 \cdot 7)\end{array}$ \\
\hline Solicited injection site adverse events & 55 & $\begin{array}{c}47 \cdot 0 \\
(37 \cdot 7-56 \cdot 5)\end{array}$ & 55 & $\begin{array}{c}47 \cdot 4 \\
(38 \cdot 1-56 \cdot 9)\end{array}$ & $\begin{array}{c}-0 \cdot 4 \\
(-13 \cdot 4-12 \cdot 7)\end{array}$ \\
\hline Pain & 18 & $\begin{array}{c}15 \cdot 4 \\
(9 \cdot 4-23 \cdot 2)\end{array}$ & 13 & $\begin{array}{c}11 \cdot 2 \\
(6 \cdot 1-18 \cdot 4)\end{array}$ & $\begin{array}{c}4 \cdot 2 \\
(-8 \cdot 5-17 \cdot 0)\end{array}$ \\
\hline Redness & 45 & $\begin{array}{c}38 \cdot 5 \\
(29 \cdot 6-47 \cdot 9)\end{array}$ & 47 & $\begin{array}{c}40 \cdot 5 \\
(31.5-50.0)\end{array}$ & $\begin{array}{c}-2 \cdot 0 \\
(-14 \cdot 6-11 \cdot 1)\end{array}$ \\
\hline Swelling & 0 & $\begin{array}{c}0 \\
(0 \cdot 0-3 \cdot 1)\end{array}$ & 3 & $\begin{array}{c}2 \cdot 6 \\
(0 \cdot 5-7 \cdot 4)\end{array}$ & $\begin{array}{c}-2 \cdot 6 \\
(-15 \cdot 3-10 \cdot 2)\end{array}$ \\
\hline Induration & 3 & $\begin{array}{c}2 \cdot 6 \\
(0 \cdot 5-7 \cdot 3)\end{array}$ & 5 & $\begin{array}{c}4 \cdot 3 \\
(1 \cdot 4-9 \cdot 8)\end{array}$ & $\begin{array}{c}-1 \cdot 7 \\
(-14 \cdot 5-11 \cdot 1)\end{array}$ \\
\hline Grade 3 solicited injection site adverse event & 0 & $\begin{array}{c}0 \\
(0 \cdot 0-3 \cdot 1)\end{array}$ & 1 & $\begin{array}{c}0 \cdot 9 \\
(0 \cdot 0-4 \cdot 7)\end{array}$ & $\begin{array}{c}-0 \cdot 9 \\
(-13 \cdot 6-11 \cdot 9)\end{array}$ \\
\hline Any unsolicited adverse event within 4 weeks after vaccination with mIPV2HD/IPV & 53 & $\begin{array}{c}45 \cdot 3 \\
(36 \cdot 1-54 \cdot 8)\end{array}$ & 58 & $\begin{array}{c}50 \cdot 0 \\
(40 \cdot 6-59 \cdot 4)\end{array}$ & $\begin{array}{c}-4 \cdot 7 \\
(-17 \cdot 6-8 \cdot 4)\end{array}$ \\
\hline Related to vaccine & 1 & $\begin{array}{c}0 \cdot 9 \\
(0 \cdot 0-4 \cdot 7)\end{array}$ & 0 & $\begin{array}{c}0 \\
(0 \cdot 0-3 \cdot 1)\end{array}$ & $\begin{array}{c}0 \cdot 9 \\
(-11 \cdot 9-13 \cdot 6)\end{array}$ \\
\hline Any SAE during the 34-week study period & 8 & $\begin{array}{c}6 \cdot 8 \\
(3 \cdot 0-13 \cdot 0)\end{array}$ & 15 & $\begin{array}{c}12 \cdot 9 \\
(7 \cdot 4-20 \cdot 4)\end{array}$ & $\begin{array}{c}-6 \cdot 1 \\
(-18 \cdot 7-6 \cdot 8)\end{array}$ \\
\hline SAE within 8 weeks after vaccination with mIPV2HD/IPV & 6 & $\begin{array}{c}5 \cdot 1 \\
(1 \cdot 9-10 \cdot 8)\end{array}$ & 7 & $\begin{array}{c}6 \cdot 0 \\
(2 \cdot 5-12 \cdot 0)\end{array}$ & $\begin{array}{c}-0 \cdot 9 \\
(-13 \cdot 6-11 \cdot 9)\end{array}$ \\
\hline Any important medical event during the 34-week study period & 1 & $\begin{array}{c}0 \cdot 9 \\
(0 \cdot 0-4 \cdot 7)\end{array}$ & 1 & $\begin{array}{c}0 \cdot 9 \\
(0 \cdot 0-4 \cdot 7)\end{array}$ & $\begin{array}{c}0 \\
(-12 \cdot 8-12 \cdot 8)\end{array}$ \\
\hline
\end{tabular}




\begin{tabular}{|c|c|c|c|c|c|}
\hline & \multicolumn{2}{|c|}{ mIPV2HD group } & \multicolumn{2}{|c|}{ IPV group } & \multirow{2}{*}{ 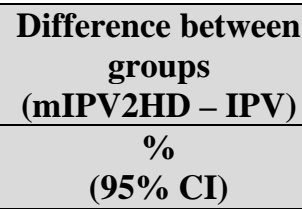 } \\
\hline & $\mathbf{n}$ & $\begin{array}{c}\% \\
\text { (95\% CI) }\end{array}$ & $\mathbf{n}$ & $\begin{array}{c}\% \\
(95 \% \mathrm{CI})\end{array}$ & \\
\hline Any important medical event within 8 weeks after vaccination with mIPV2HD/IPV & 0 & $\begin{array}{c}0 \\
(0 \cdot 0-3 \cdot 1)\end{array}$ & 0 & $\begin{array}{c}0 \\
(0 \cdot 0-4 \cdot 7)\end{array}$ & $\begin{array}{c}0 \\
\text { (NA) }\end{array}$ \\
\hline
\end{tabular}

mIPV2HD = monovalent inactivated poliovirus vaccine, type 2, high dose. IPV = trivalent inactivated poliovirus vaccine. 95\% CI = Exact 95\% confidence interval.

The following vaccines were administered concomitantly with polio vaccines:

with bOPV (dose 1) at 6 weeks of age; diphtheria - tetanus - whole cell pertussis - hepatitis B - Haemophilus influenzae type b (DTPw-HepB-Hib) vaccine (or DTPw-Hib vaccine), pneumococcal vaccine, and rotavirus vaccine. Infants who received DTPw-Hib vaccine at Day 0 were vaccinated with hepatitis B vaccine at 10 weeks of age with bOPV (dose 2).

with mIPV2HD/IPV at 14 weeks of age: bOPV (dose 3) and rotavirus vaccine with mOPV2 challenge at 18 weeks of age: DTPw-HepB-Hib vaccine and pneumococcal vaccine.

A third dose of DTPw-HepB-Hib vaccine was administered at 24 weeks of age; influenza vaccine was administered at 24 and 28 weeks of age. 
APPENDIX 
Figure 2: Reverse cumulative distribution of type 2 poliovirus shedding index after mOPV2 challenge

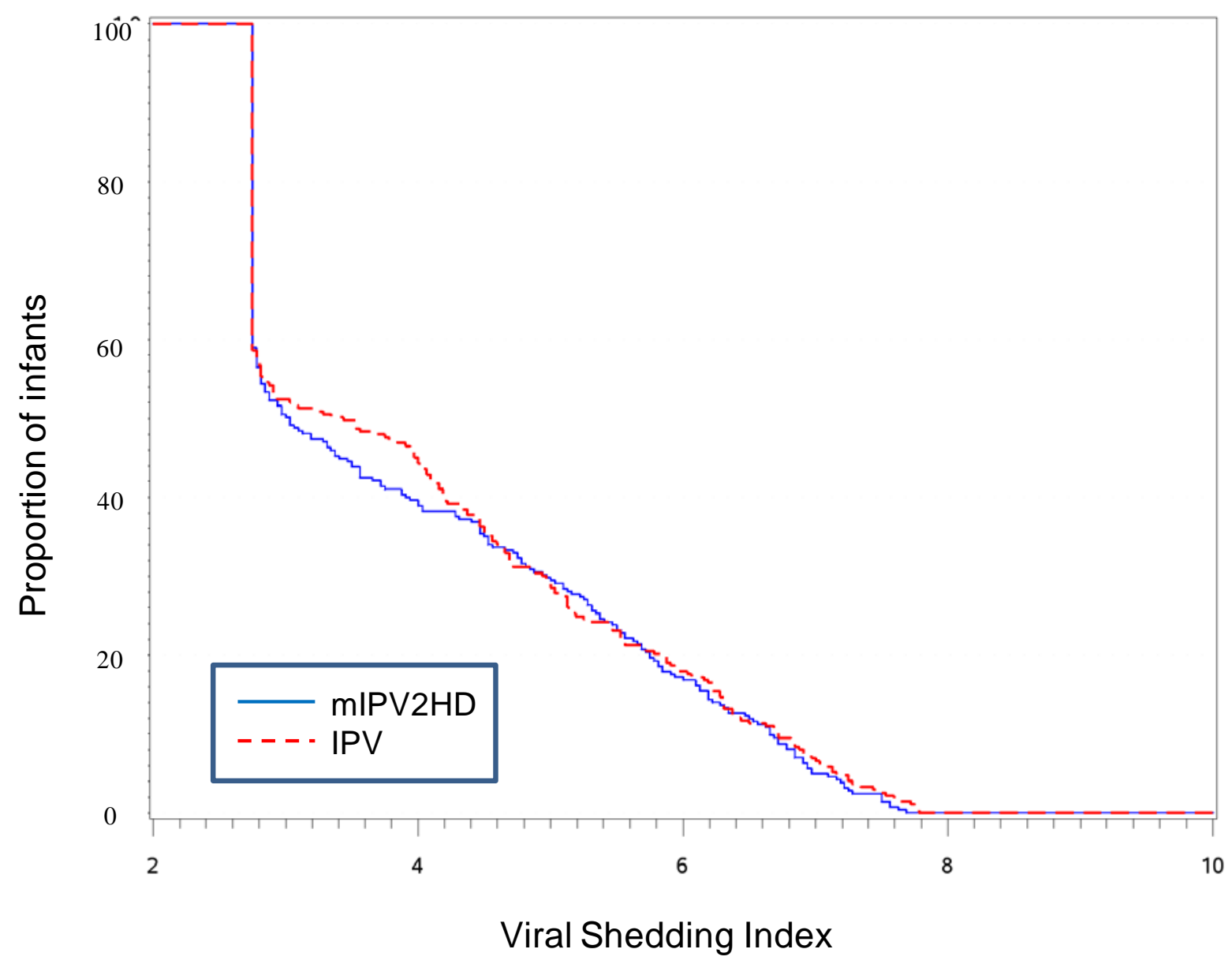

